\title{
Les villes du pouvoir et le réseau des grandes voies romaines de l' Hispanie
}

Pierre Sillières

Centre Ausonius, Bordeaux

p.sillieres@wanadoo.fr

Fecha recepción 22.02.2016 / Fecha aceptación 05.05.2016

\section{Resume}

Toutes les villes du pouvoir de l' Hispanie, capitales provinciales et chefs-lieux de conventus, se trouvaient sur les grands axes routiers de la péninsule, les viae militares parcourues par les voitures du cursus publicus. Quant aux chantiers de construction et d'entretien des chaussées, on sait que la plupart étaient réalisés dans le cadre de la province et, au niveau local, dans celui de la cité. Mais, les chefs-lieux de conventus étant de véritables centres régionaux, dans lesquels devaient fréquemment se rendre les populations de leur circonscription, il paraît également très vraisemblable qu' un bureau chargé de leurs voies existait dans ces grandes villes, comme semblent l' indiquer des textes de plusieurs bornes milliaires des conventus de Galice ou de Clunia.

\section{Mots-cles}

Conventus, cursus publicus, via militaris, milliaire, voie Bracara Augusta-Lucus Augusti, voie TuriasoClunia

\section{Abstract}

In Hispania, all cities of power, i.e., the provincial capitals and the main conventus cities, were on the main roads of the peninsula, the viae militares, which were used by the cursus publicus. As regards construction sites and road maintenance, we know that most of them were to be found locally within the province, i.e., in the city. But the chief conventus cities were genuine regional centres, which were often where the people of the land subdivision would meet, so it also seems very likely that an office responsible for the roads existed within these cities, as is suggested by texts on several milestones of the Galician conventus and Clunia.

\section{Key words}

Conventus, cursus publicus, via militaris, milliaire, road Bracara Augusta-Lucus Augusti, road TuriasoClunia 
En Hispanie, comme dans le reste de l'Empire, le pouvoir romain s'exerçait par le relais des capitales provinciales, Tarraco, Corduba et Emerita, où résidaient les gouverneurs, représentants de l'empereur, les légats, les procurateurs et le personnel des bureaux. Mais les provinces hispaniques ont, en outre, été dotées de circonscriptions supplémentaires, celles des conventus que seules l'Asie et la Dalmatie connurent aussi. Dans les onze grandes villes, chefs-lieux de ces conventus hispaniques, se sont également créés progressivement des rouages administratifs nouveaux avec quelques fonctionnaires. C'est sur cet ensemble de villes du pouvoir, capitales provinciales et chefs-lieux de conventus, que reposait la mâ̂trise par Rome de l'espace provincial.

Les relais supplémentaires que représentèrent les conventus ont quelque peu amélioré l'administration des vastes et lointains espaces hispaniques qui ont ainsi été mieux et plus étroitement liés à Rome et à l'empereur. Cette nouvelle proximité entre les provinciaux et le pouvoir romain se manifestait principalement lors de la venue au chef-lieu du conventus du représentant de l'empereur, le gouverneur, qui, périodiquement, s'y installait quelque temps afin d' y organiser les assises judiciaires et qui y jugeait au nom de César Auguste. Elle apparaissait aussi lors des réunions du conseil de conventus constitué par les délégués des cités. Mais elle s'exprimait encore, peut-être davantage d'ailleurs, lors des cérémonies religieuses du culte impérial que célébrait le prêtre du conventus et auxquelles étaient associés les cités et les citoyens de la circonscription.

Enfin le ciment entre les provinces hispaniques et l'empereur, peut-être le meilleur, fut le service de transmission des ordres et des nouvelles, le cursus publicus instauré par Auguste, ainsi que son complément indispensable, le réseau de bonnes chaussées qui se constitua peu à peu pour assurer la circulation des voitures. En effet, le bon fonctionnement de cette poste impériale a reposé sur l'aménagement progressif et l'entretien de bonnes routes, les viae militares, qui relièrent toutes les villes du pouvoir de l'espace péninsulaire et facilitèrent considérablement les relations entre elles et avec Rome. Ciment d'autant plus efficace que son fondateur ne chercha pas seulement à créer un service de transmission des dépêches et de transport des fonctionnaires, mais voulut aussi constituer 
en même temps un organe de renseignement sur les Hispani et de contrôle des administrations provinciales et locales de la péninsule : à cet effet, il avait prévu que le messager qui, grâce au transport en voiture avait effectué tout le voyage depuis l' Espagne, pût être interrogé sur l'état de la région dont il arrivait ${ }^{1}$.

\section{I . Les chefs-lieux de conventus et les viae militares de I' Hispanie}

La position de ces grandes villes du pouvoir sur les grands axes du cursus publicus facilita assurément ce contrôle. Pour mettre en évidence la situation de ces villes sur le réseau des principales chaussées des provinces hispaniques, celles qu'empruntaient les voitures de la poste impériale, il faut parvenir à en reconnaître les trajets. A première vue ce n'est pas simple car il paraît compliqué de distinguer ces grands axes de la circulation en Hispanie du reste des nombreuses voies que mentionnent les itinéraires antiques, l'Itinéraire d'Antonin et l'Anonyme de Ravenne principalement ${ }^{2}$, sans faire la moindre distinction à leur propos. Aussi les cartes à notre disposition ne s'y sont pas risquées. Pourtant cette démarche est indispensable, même si elle n' est guère aisée : chercher à établir une hiérarchie des différents voies, c'est-à-dire à réaliser une représentation cartographique de la circulation dans les provinces hispaniques est d' un intérêt primordial pour tous les domaines d' investigation, tant politiques qu'économiques. Ce classement ne peut être fondé que sur quelques indications d'auteurs antiques et, surtout, sur l' apport de la documentation archéologique représentée principalement par les trouvailles de milliaires et les vestiges de chaussées antiques.

1. Le texte concis mais extrêmement précis de Suétone, Aug., 49.5, résume parfaitement le dessein d'Auguste : «Et pour que pût être annoncé et connu plus vite et facilement ce qui se passait dans chaque province, il disposa d'abord des jeunes gens, ensuite des voitures à des distances moyennes le long des grandes voies impériales (viae militares). Cette dernière mesure parut plus pratique, "afin que ceux qui en personne portent les lettres du lieu de départ jusqu'à la fin du parcours, pussent encore être interrogées si les circonstances le réclamaient en quoi que ce soit’" trad. H.G. Pflaum, Essai sur le Cursus publicus sous le HautEmpire romain (MAI, 14), Paris, 1940, 23, sauf pour viae militares qui ne sont pas nécessairement des «voies militairement occupées» mais simplement les grandes artères de l'Empire ; à ce propos voir R. Rebuffat, «Via militaris», Latomus, 1987, 52-67, P. Sillières, Les voies de communication de l' Hispanie méridionale, Bordeaux 1990 (=Les voies de communication...), 785-790, et plus récemment M.A. Speidel, «Heer und Strasse - militares viae», in R. Frei-Stolba (éd.), Siedlung und Verkehr im römischen Reich. Römerstrassen zwischen Herrschaftssicherung und Landschaftsprägung, Berne 2004, 331-344.

2. L’ensemble de ces sources a été rassemblé par J.M. Roldán Hervás, Itineraria Hispana. Fuentes antiguas para el estudio de las vías romanas en la península Ibérica (Supplément de Hispania Antiqua), ValladolidGranada 1975. Voir aussi G. Arias Bonet, Repertorio de caminos de la Hispania romana, Ronda 2004, 2a ed., et J.M. Roldán Hervás y C. Caballero Casado, Itinera Hispana. Estudio de las vías romanas de Hispania a partir del Itinerario de Antonino, el Anónimo de Rávena y los Vasos de Vicarello, El Nuevo Miliario, $\mathrm{n}^{\circ} 17$ Monográfico, Madrid 2014. 
Monográfico | Les villes du pouvoir et le réseau des grandes voies romaines de l' Hispanie

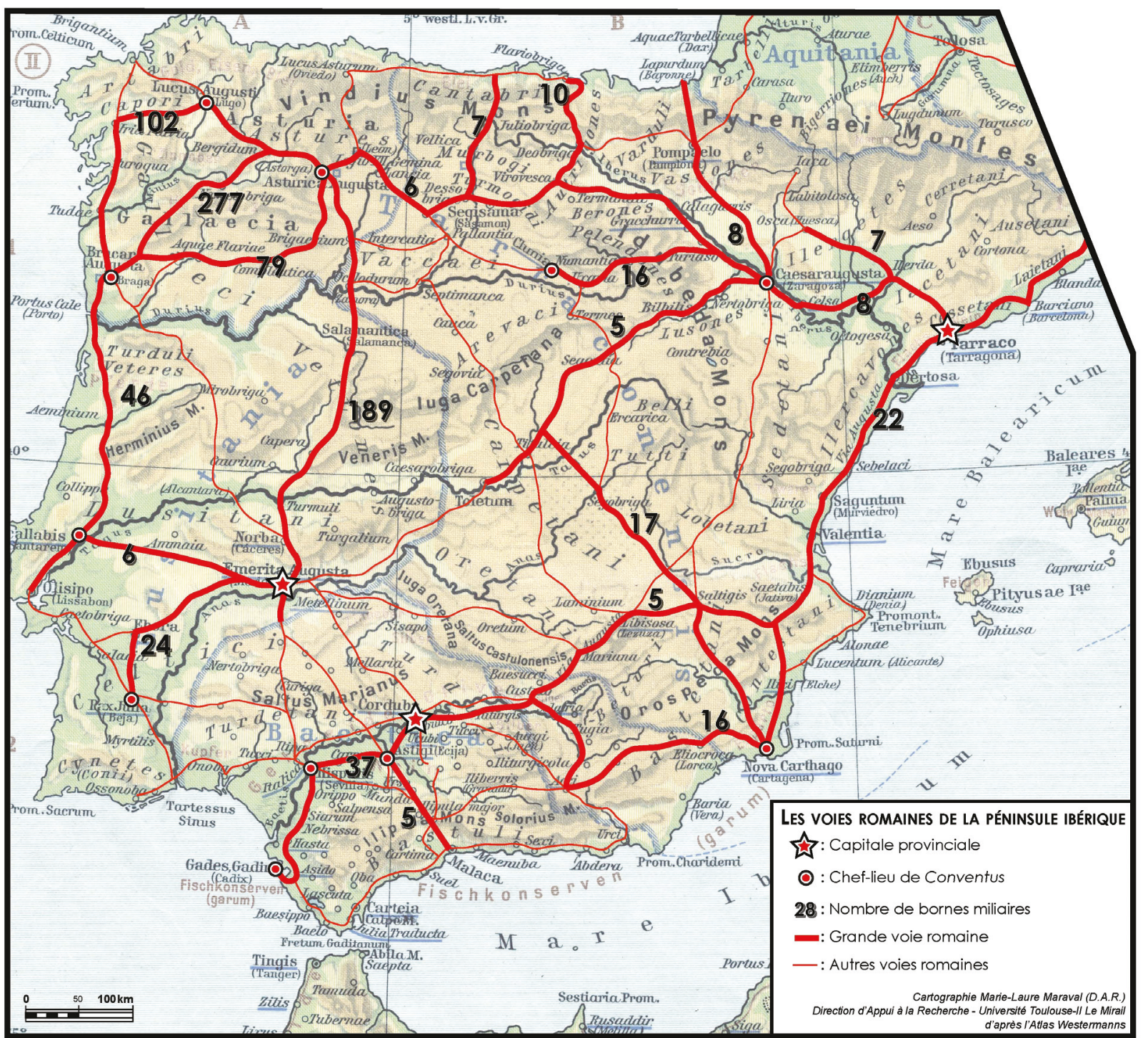

Fig. 1 : Les villes du pouvoir et les principales voies romaines (viae militares) de l' Hispanie romaine 


\section{Les grands axes de la circulation dans la péninsule lbérique et leur cons- truction au début de l' époque impériale (fig. 1)}

Cette carte, qui est presque totalement dépendante de l'état des trouvailles de milliaires et des fouilles sur les chaussées, présente sans aucun doute des lacunes et des erreurs. Aussi devrat-on l' améliorer progressivement à mesure de l' avancement des connaissances. Toutefois, les éléments qui étayent le trajet de chacun de ses itinéraires, principalement les milliaires et les restes de voies, en général concordent.

\section{a. En Bétique}

Pour la province méridionale de l' Hispanie, la Via Augusta, doit être retenue en priorité. En effet, non seulement sa chaussée apparaît sur une grande partie de son trajet $^{3}$, mais elle a aussi livré un assez grand nombre de milliaires entre le Ianus Augustus, à son entrée dans la province, et Gades, 37 au dernier recensement dont 9 d'époque augustéenne ${ }^{4}$. Elle conserve en outre les ponts antiques de Vila del Río, de l'Arroyo de Pedroche, de Cordoue, du Puente Viejo sur le Guadajoz et d'Alcantarillas construit sans doute sous Auguste ${ }^{5}$.

Ensuite, un deuxième itinéraire peut aussi être considéré comme un axe important de la province, au moins à partir du règne de Domitien. C'est la voie qui reliait Astigi (Écija) à Malaca (Málaga) par Ostippo (Estepa) et Anticaria (Antequera); en effet, aux vestiges de la chaussée reconnus à La Marinaleda, au sud d' Écija, et aux 4 bornes que nous lui avions attribuées ${ }^{6}$, s'est ajouté récemment le milliaire d'Antequera, érigé en 87 , qui mentionne la construction de la Via Domitiana Augusta à cette date?.

\section{b. En Lusitanie}

Les chaussées importantes paraissent avoir été plus nombreuses dans l'ouest de la péninsule. L' une d'elles, la voie Emerita (Mérida)-Salmantica (Salamanque), mieux connue sous son nom médiéval de Vía de la Plata, l'emporte très nettement car elle constituait l' artère principale de la province. À notre connaissance, au moins 189 bornes ont été découvertes sur son trajet $^{8}$, la majorité érigée sous Trajan et Hadrien mais deux sont attribuables à Auguste et un

3. Comme l' indique la carte des voies de l' Hispanie méridionale, P. Sillières, Les voies de communication..., in fine.

4. P. Sillières, Les voies de communication..., op. cit. $\mathrm{n}^{\text {os }} 22$ à 56.

5. P. Sillières, Les voies de communication..., op. cit.659-691.

6. P. Sillières, Les voies de communication..., op. cit. $\mathrm{n}^{\text {os }} 71$ à 74 .

7. A.U. Stylow, R. Atencia Páez et J.C. Vera, “ Via Domitiana Augusta ”, Mainake, 26, 2004, 417-430: ... Dom[itianus Aug] ....viam fecit Domitianam Augustam.

8. J.M. Roldán Hervás, Iter ab Emerita Asturicam. El camino de la Plata. Memorias del Seminario de Prehistoria y Arqueología de la Universidad de Salamanca, n 3, Salamanca, 1971, 129-150 et pl. I-XXXVI ; 
Tibère9 ${ }^{9}$ Elle conserve aussi les ponts de Mérida, de l'Albarregas, d'Aljucén, d'Alconetar, de Caparra et de Salamanque ${ }^{10}$ et sa chaussée a été reconnue et suivie sur presque tout son trajet, entre la frontière avec la Bétique au sud, près de Villafranca de los Barros, et, au nord, avec celle de l' Espagne Citérieure, située à une trentaine de km au-delà du Río Tormes ${ }^{11}$.

Ensuite, une seconde grande voie, également de direction nord-sud, longeait le littoral océanique entre Olisipo (Lisbonne) et Bracara Augusta (Braga) ; 46 milliaires en proviennent, le plus ancien érigé au nom d'Auguste. Deux autres axes étaient aussi aménagés en grande voie romaine, tous deux menant à la capitale provinciale Emerita (Mérida) : l' un depuis Pax Iulia (Beja) pour lequel on connaît 24 bornes, dont au moins une quinzaine entre Ebora (Evora) et Pax Iulia, l' autre depuis Scallabis (Santarem) sur lequel ont été découverts 6 milliaires et qui conserve le très beau pont de Vila Formosa ${ }^{12}$.

\section{c. En Espagne Citérieure}

La plus vaste province de l' Hispanie a disposé du réseau le plus complet de grandes chaussées impériales. Cet ensemble s'organise selon trois directions. L' axe principal est la grande rocade nord-sud/sud-ouest, constituée par la Via Augusta et le Camino de Aníbal, qui fut empruntée très tôt par les Romains, dès de la Seconde Guerre Punique et, aménagée précocement aussi, peut-être, à en croire Polybe, vers le milieu du IIe s. av. J.-C. ${ }^{13}$. Sur son trajet longeant le littoral, bien établi aujourd' hui, 22 bornes ont été découvertes entre le Summum Pyrenaeum (Col de Panissar) et Carthago Nova (Carthagène) parmi lesquelles 3 sont d'époque augustéenne comme le pont qui se conserve à Martorell ; puis 16 autres, dont 6 au nom d'Auguste entre Carthago Nova et la frontière de la Bétique près de Castulo. Enfin le Camino de Aníbal, qui était le seul itinéraire entre la Méditerranée et la vallée du Guadalquivir à l'époque républicaine $^{14}$, a continué à être emprunté à l'époque impériale, sans doute parce qu' il était nettement plus court que le Via Augusta; il a livré 5 bornes milliaires et sa chaussée est encore visible en de nombreux endroits.

C. Puerta Torres, Los miliarios de la Via de la Plata, thèse de l’Université Complutense, Madrid, 1995, 23-30; le milliaire le plus septentrional a été trouvé à Calzada de Valdunciel, $15 \mathrm{~km}$ au-delà de Salamanque.

9. C. Puerta Torres, Los miliarios... op. cit.

10. M. Durán Fuentes, La constucción de puentes romanos en Hispania, Saint-Jacques de Compostelle, 2005.

11. Pour cette frontière de la Lusitanie voir J. Alarcão et alii, “ Proposition pour un nouveau tracé des limites anciennes de la Lusitanie romaine ”, in : Les villes de la Lusitanie romaine (Talence, 1988), Paris, 1990, 319-329 et carte in fine.

12. M. Durán Fuentes, La constucción de puentes... op. cit., 162-169; P. Sillières " Les ponts romains de la péninsule Ibérique. Chronologie et approche des techniques architecturales ”, in : G. Barruol, G., J.-L. Fiches et P. Garmy (dir.), Les ponts routiers en Gaule romaine, RAN, suppl. 41, Montpellier, 2011, 645.

13. P. Sillières “ De Polybe, III, 3, aux Gobelets de Vicarello : la voie de l'Ebre au Rhône à l'époque républicaine ”, in : M. Bats et alii (éd.), Peuples et territoires en Gaule méditerranéenne. Hommage à Guy Barruol, RAN, suppl. 35, Montpellier, 2003, 121-127.

14. Cic., Fam. X, 31 ; P. Sillières, Les voies de communication..., op. cit. 566-568. 
Le deuxième ensemble de voies, dont les itinéraires furent aussi empruntés très tôt par les Romains lors des principales campagnes militaires du $\mathrm{II}^{\mathrm{e}}$ et du $\mathrm{I}^{\mathrm{er}}$ s. av. J.-C., avait une orientation est-ouest et son axe principal était la chaussée de la vallée de l'Ebre, depuis Tarraco (Tarragone), par Caesaraugusta (Sarragosse) et jusqu' à Asturica Augusta. Plusieurs routes importantes en divergeaient : une à partir d' Ilerda (Lérida) menait à Osca (Huesca); trois à partir de Caesaraugusta, l' une allant l' une jusqu'à Oiasso (Irún) par Pompaelo (Pampelune), l' autre jusqu' à Toletum (Tolède) par Bilbilis et Complutum (Alcalá de Henares); la troisième jusqu' à Clunia par Turiaso (Tarazona); enfin trois depuis Asturica Augusta, toutes aboutissant à Bracara Augusta par des trajets différents. Hormis quelques premiers travaux routiers effectués dès l'époque républicaine entre Ilerda et Celsa, notamment la construction du pont de Celsa ${ }^{15}$, toutes ces grandes voies furent aménagées dès le début de l' Empire, chantiers datés par 21 milliaires d'Auguste et 17 de Tibère.

Enfin, dans une troisième direction s'orientait la voie Carthago Nova (Carthagène)Complutum (Alcalá de Henares) dont d' importants vestiges de la chaussé se conservent entre sur plusieurs tronçons de son trajet ${ }^{16}$. Elle a aussi été réalisée dès le début de l'époque impériale puisque une borne d'Auguste et six de Tibère ont été découvertes sur son trajet ${ }^{17}$.

\section{Les villes du pouvoir et le réseau de ces grandes voies}

Grâce à cette carte, même provisoire, apparaît clairement la place des capitales provinciales et des chefs-lieux de conventus dans la circulation routière en Hispanie à l'époque impériale. On y constate d'emblée que ce réseau des grandes chaussées s'organisait à partir des deux villes qui peuvent être considérées comme les capita viarum des province hispaniques, Tarraco d'abord, Carthago Nova ensuite. Cette primauté tient sans doute à leur rôle dans l' histoire de l' implantation romaine dans la péninsule et remonte aux premiers temps de la conquête, mais elle s'explique surtout par leur situation extrêmement favorable pour les relations avec Rome et l' Italie. Il y apparait aussi parfaitement que toutes les villes du pouvoir se trouvaient sur ces grands axes routiers, les viae militares parcourues par les voitures du cursus publicus.

En Bétique, les quatre grandes villes, Corduba (Cordoue) la capitale provinciale, et les chefs-lieux de conventus Astigi (Écija), Hispalis (Séville) et Gades (Cadix) jalonnaient la Via Augusta. Ajoutons que Corduba et Hispalis étaient en outre baignées par le Guadalquivir et Astigi par le Genil, fleuves animés d' une très active navigation fluviale.

15. Strab., III, 4, 10.

16. L'étude la plus précise du point de vue de la localisation des vestiges vient d'être proposée par J.L. Fernández Montoro, J. Lostal Pros et J. Rodríguez Morales, “ La calzada romana Carthago-Nova a Complutum : síntesis de su recorrido”, El Nuevo miliario, 13, 2011, 32-54.

17. Deux de celles-ci, trouvées récemment à Huelves et érigées entre 26 et 32, indiquent la distance de 221 milles jusqu' à Carthago Nova, le caput viae : J.M. Abascal et R. Cebrián, “ Carthago Nova como caput viae. Dos miliarios de Tiberio de Huelves (Hispania citerior) ”, ZPE, 162, 2007, 257-262. 
En Lusitanie, le centre du réseau routier était Emerita Augusta, la capitale provinciale, et les deux chef-lieux de conventus, Scallabis (Santarem) et Pax Iulia (Beja), étaient reliés à celle-ci par deux bonnes chaussées.

En Espagne citérieure, Tarraco et Carthago Nova étaient toutes deux sur la Via Augusta du littoral tandis que Caesaraugusta et Asturica Augusta, cités de l' intérieur, se trouvaient au milieu et à l'extrémité occidentale de la grande route de la vallée de l'Ebre. Ces deux chefslieux de conventus avaient probablement une certaine prééminence par rapport aux autres grandes villes de cette partie nord de la province car ils occupaient un point de convergence de plusieurs grandes voies. Les deux autres chefs-lieux de conventus du Nord-Ouest se trouvaient, d'après le nombre des bornes milliaires découvertes, sur les voies les mieux entretenues de la péninsule : Lucus Augusti (Lugo) était située sur la grande rocade occidentale, et Bracara Augusta (Braga) profitait sans doute de sa situation de carrefour, à l' aboutissement des trois importantes chaussées de la Galice et à leur jonction avec la voie lusitanienne venant de Scallabis (Santarém).

Toujours à propos des villes du pouvoir de l' Espagne citérieure, le cas de Clunia mérite quelque attention supplémentaire car il éclaire la relation entre une grande voie et un cheflieu de conventus. L'étude précise du trajet de la route romaine entre Uxama et Augustobriga ${ }^{18}$ a permis de constater qu' elle présentait encore à la fin du XIX ${ }^{\mathrm{e}} \mathrm{s}$. les vestiges d' une chaussée bien construite et un bon nombre de bornes milliaires, 16 au total, dont 3 de Tibère ; elle avait donc été ouverte dés le début du $\mathrm{I}^{\mathrm{er}} \mathrm{s}$. et bien entretenue par la suite, surtout au $\mathrm{II}^{\mathrm{e}} \mathrm{s}$. Mais il est aussi apparu, au cours de recherches postérieures, que ces caractéristiques des viae militares disparaissent au-delà de Clunia. On a donc l' impression que cet itinéraire n'a été aménagé en grande voie à chaussée épaisse que jusqu' à Clunia, c'est-à-dire que l'autorité romaine qui a décidé de sa construction et, par la suite, de son entretien ne s'est intéressé qu' au trajet menant au chef-lieu de conventus.

\section{Les communications à l' intérieur des conventus}

Les chefs-lieux de conventus étaient de véritables centres régionaux et les populations des cités appartenant à leur circonscription s'y rendaient fréquemment. Ces voyages s' effectuaient, non seulement pour des raisons administratives et politiques, à l'occasion des sessions de justice, des réunions du conseil du conventus ou de cérémonies du culte impérial, mais encore et sans doute beaucoup plus souvent dans le cadre de relations économiques. Ils correspondaient en effet à des villes importantes, presque toujours des colonies romaines, sauf pour les trois créations augustéennes du Nord-Ouest, et à des centres économiques très actifs avec commerces et marchés.

Pour ces déplacements à l' intérieur du territoire du conventus pouvaient, en premier lieu, être empruntées les grandes voies romaines que l'on vient de présenter. Les cas les plus favorables pour ces communications régionales correspondaient aux conventus dans lesquel

18. E. Saavedra, Descripción de la vía romana entre Uxama y Augustobriga, Madrid, 1861. 
le chef-lieu occupait une position centrale. Le meilleur exemple est celui de Caesaraugusta (Saragosse) où se rejoignaient de grandes routes provenant de toutes les directions : de l'est celle d' Ilerda et Tarraco, de l'ouest celle de Gracchuris et d' Asturica, du nord-ouest celle de Pompaelo et d' Oiasso et du sud celle de Bilbilis et Toletum. Á celles-ci, s'ajoutaient les routes plus médiocres d'Osca, de Iacca, et de Contrebia indiquées par les itinéraires antiques et, surtout, le grande artère fluviale de l' Èbre, navigable en aval comme en amont de la ville. Ce réseau extrêmement dense est probablement la raison principale de l' importance de la cité de Caesaraugusta dans la province d' Espagne citérieure.

Carthago Nova, Hispalis, Scallabis et Asturica Augusta correspondent à d'autres cas assez comparables et des conclusions similaires s' imposent à leur propos. Bien sûr Carthago Nova constitue un cas particulier du fait de son passé prestigieux, de sa fonction de grand port méditerranéen et de la proximité des mines d'argent, mais sa position à la jonction de deux plus grandes voies romaines de l'Hispanie méridionale, la Via Augusta et la voie Carthago Nova-Complutum, a assurément conforté son importance politique et économique et sa prééminence sur tout le sud-est de la péninsule. Hispalis (Séville), qui était, elle aussi, une vieille agglomération et possédait également un grand port à la fois fluvial et maritime, se trouvait en outre au centre d' un dense réseau routier. Sans doute celui-ci ne comptait qu' une grande chaussée, la Via Augusta, mais il comportait plusieurs autres routes mentionnées par les itinéraires antiques et qui venaient d' Emerita, d' Onoba, de Salpensa, et d' Ostippo. Scallabis était aussi au carrefour de deux grandes chaussées, celle du littoral occidental entre Olisippo et Bracara et celle qui menait à Emerita. Surtout elle était desservie par la magnifique voie d'eau du Tage, accessible aux très grands bateaux et par les embarcations plus légères sur 500 stades $^{19}$. Quant à Asturica Augusta, cité bien moins illustre, elle était aussi au centre d' un excellent réseau routier constitué de bonnes chaussées constamment entretenues, les trois grandes voies du Nord-Ouest conduisant à Bracara Augusta et la Vía de la Plata menant à Emerita.

Les autres chefs-lieux sont plus excentrés dans le territoire de leur conventus. Ainsi Pax Iulia et Clunia se trouvent sur une seule grande voie et à l'extrémité de celle-ci. Toutefois elles étaient assurément desservies par d' autres routes et chemins, moins bien construits sans doute, mais suffisants pour les déplacements sur des distances assez courtes.

\section{Une administration des routes au chef-lieu de conventus?}

Ce réseau de routes et de chemins à l' intérieur de chaque conventus et cette circulation assez dense entre la ville principale et les autres cités du territoire ne réclamaient-ils pas la présence d' un service des routes dans chacune de ces circonscriptions, c'est-à-dire d' un bureau responsable de l'entretien de ses voies ? Cette question a été examinée à propos de l' Hispanie méridionale et il est apparu alors que les chantiers de construction et d'entretien des chaussées étaient seulement décidés puis réalisés dans le cadre de la province et,

19. Strab., 3.3.1. 
au niveau local, dans celui de la cité2 ${ }^{2}$. En effet, la quasi totalité de la documentation disponible atteste de façon extrêmement claire le rôle des gouverneurs provinciaux dans la construction et l'entretien des grandes voies romaines dans toutes les régions de l' Empire romain ${ }^{21}$. Pour la péninsule Ibérique, il suffit de rappeler les exemples de C. Calpetanus Rantius sous les Flaviens ${ }^{22}$, de Q. Decius sous Maximin ${ }^{23}$ et d'Antonius Maximus à la fin du IVe siècle $^{24}$. Quant à l' activité des cités dans ce domaine, elle est illustrée par plusieurs textes épigraphiques, en particulier ceux qui concernent les très grands chantiers des ponts d'Alcantara ${ }^{25}$ et de Chaves ${ }^{26}$, ou des travaux moins fameux comme ceux qui furent réalisés pour leur cité par des évergètes de Castulo $^{27}$ et d' Oreto ${ }^{28}$.

Un retour sur cette question de l'organisation des travaux routiers, à l'occasion de notre réflexion sur les conventus, $\mathrm{m}$ ' incite, cependant, à prendre maintenant à une position plus nuancée. En effet, à l'examen de l'ensemble des textes de milliaires de la péninsule Ibérique, apparaissent quelques indices qui semblent suggérer qu' existèrent sans doute dans les chefs-lieux de conventus, au moins dans quelques-uns, une petite administration chargée des routes. C'est sur les textes d' un certain nombre de bornes milliaires, les unes du Nord-Ouest, les autres de la voie Clunia-Turiaso, que se fonde cette nouvelle vision.

\section{Dans le Nord-Ouest}

Il y a déjà assez longtemps, une théorie a eu quelque succès à propos des variations des formules impériales sur les bornes du Nord-Ouest : J. Castro Nuñez ${ }^{29}$, puis M. Estefanía Álvarez ${ }^{30}$ ont émis l' hypothèse qu' un changement dans l'énoncé de la formule impériale traduisait l'appartenance des milliaires à deux voies différentes ou, pour une même voie, son passage d' un conventus à un autre. Acceptée d' abord assez généralement, elle a ensuite perdu la plupart de ses arguments avec la révision des pierres, en particulier par A. Tranoy qui a montré que plusieurs inscriptions qui avaient été retenues n'étaient pas des textes de milliaires ${ }^{31}$.

20. P. Sillières, Les voies de communication..., op. cit. 695-701.

21. Voir en particulier la documentation utilisée par T. Pekary, Untersuchungen zu den römischen Reichsstrassen, Bonn, 1968, 77-86.

22. CIL II, 4798, 4799, 4802, 4803, 4838, 4854, 6224.

23. CIL II, 4756, 4759, 4788, 4826, 4831,4834 et 4870, 4858, 4886.

24. CIL II, 4911.

25. CIL II, 437=ILS, 287.

26. CIL II, 2478.

27. CIL II, 3270 .

28. CIL II, $3221=6339$.

29. J. Castro Núñez, “Os miliarios de Nerva na Gallaecia ”, CEG, 16, 1950, 161-174.

30. M.D.N. Estefanía Álvarez, "Notas para la delimitación de los conventus jurídicos en España ”, Zephyrus, 9, 1958, 51-57.

31. A.Tranoy, “Le pseudo-milliaire de S. Claudio (Gostei, Bragança) et les limites orientales du conventus Bracarum ", TAE, 23, 1980, 589-594. 
Toutefois, ce même historien de la Galice romaine a repris cette proposition, mais cette fois en l'étayant solidement avec une documentation parfaitement recevable. Il se fonde seulement sur les miliaires d' Hadrien appartenant à la voie Bracara Augusta-Lucus Augusti par le littoral de la Galice ${ }^{32}$, et il constate que les formules impériales de cet empereur sont différentes au nord et au sud du Río Verdugo ${ }^{33}$. Trois milliaires, qui appartiennent au tronçon au sud du Verdugo, présentent une formule au datif et indiquent une distance mesurée depuis Braga $^{34}$. Voici le texte de l' un de ces trois milliaires découverts entre le Río Verdugo et Bracara Augusta: IMP CAES TRAIANO / HADRIANO AVG /PONTIF MAX / TRIB POTEST XVIII / COS III PP A BRACAR /AVG M P XX ${ }^{35}$.

En revanche trois autres bornes, qui proviennent de la partie de la voie au nord de la rivière, portent un texte au nominatif et une distance calculée depuis Lugo ${ }^{36}$.

Le texte est le suivant : IMP CAES DIVI TRAI/ANI PARTICI FIL / DIVI NERVAE NEPOS / TRAIANVS HADRIA/NVS AVG PP PONT / MAX TRIB POT XVIII / COS III A L AUG M P / LXXXXVI ${ }^{37}$.

Pourtant les six pierres ont été érigées la même année 133. Il paraît donc tout à fait légitime d'en conclure, comme le fait A. Tranoy, que le Río Verdugo constituait la frontière entre les conventus de Lucus Augusti et de Bracara Augusta ${ }^{38}$.

Ces observations intéressent la question qui nous occupe. En effet, il est également permis de penser que si des formules impériales différentes ont été gravées au nord et au sud du Verdugo, qui était très probablement la frontière entre les deux conventus voisins, il est très vraisemblable qu'elles furent établies dans les chefs-lieux, les unes à Lucus Augusti, les autres à Bracara Augusta, et non à Tarraco par le service du gouverneur de la province. Aussi paraît-il quasiment nécessaire d'en conclure qu' il existait un bureau de l'administration routière dans chacune de ces deux villes du pouvoir. Ajoutons une autre particularité de ces deux séries de textes qui paraît encore renforcer cette conclusion : les distances indiquées furent mesurées à partir du chef-lieu du conventus, depuis Lucus Augusti au nord du Mondego, depuis Bracara Augusta au sud de la rivière. Le jalonnement, qui fut effectué à partir de ces deux villes, a donc probablement été décidé par une autorité qui résidait dans chacune d'elles.

32. It. Ant., 429,5-430,8.

33. A. Tranoy, La Galice romaine. Recherches sur le nord-ouest de la Péninsule ibérique dans l'Antiquité, Bordeaux, 1981, 161 et carte XIX.

34. A. Rodríguez Colmenero, S. Ferrer Sierra, R.A. Álvarez Asorey, Miliarios e outras inscicións viarias romanas no Noroeste hispanico (conventus Bracarense, Lucense e Asturicense), Lugo 2004, bornes $n^{\circ} 138,151$ et 190 .

35. A. Rodríguez Colmenero, S. Ferrer Sierra, R.A. Álvarez Asorey, bornes nº 151.

36. A. Rodríguez Colmenero, S. Ferrer Sierra, R.A. Álvarez Asorey, bornes n 195, 200 et 201.

37. A. Rodríguez Colmenero, S. Ferrer Sierra, R.A. Álvarez Asorey, bornes nº 200.

38. A. Tranoy, La Galice romaine..., op. cit. 161. 


\section{Dans le conventus de Clunia : les milliaires de la voie Turiaso-Clunia}

Cette route qui menait à Clunia depuis la vallée de l' Ebre était assez bien conservée à la fin du $\mathrm{XIX}^{\mathrm{e}}$ s., en particulier entre Augustobriga (Muro de Agreda) et Uxama (Burgo de Osma). Ce trajet a fait l'objet à ce moment-là d' une étude qui peut toujours être prise comme modèle, en particulier pour la carte à grande échelle qui l'accompagne et les figures qui représentent les vestiges de la chaussée qui étaient encore visibles ${ }^{39}$.

Sur les 16 bornes milliaires découvertes sur le trajet de cette grande chaussée romaine, la plupart portent une indication de distance et c'est presque toujours à partir d'Augustobriga que celle-ci a été mesurée. Deux des milliaires de Tibère, qui correspondent au jalonnement le plus ancien, peut-être le premier, portent déjà Augustobriga pour caput viae ${ }^{40}$, comme la série la plus complète, constituée par les 5 bornes de Trajan ${ }^{41}$. Cette ville était donc le caput viae de cet itinéraire et le jalonnement par les bornes avait commencé à cet endroit dès l' aménagement de la chaussée terminé vraisemblablement sous Tibère.

Comment expliquer le choix de ce caput viae? On sait que les capita viarum étaient souvent une ville, d' importance variable d'ailleurs, par exemple pour la Galice Bracara Augusta, Lucus Augusti, Aquae Flaviae, Tudae et même le camp romain et relais routier de Petavonium. Mais ils coïncidaient très fréquemment aussi avec une limite territoriale, une frontière provinciale le plus souvent ${ }^{42}$ mais aussi de conventus, comme on vient de le voir à propos de bornes de la voie de Lugo à Braga, ou encore de cité ${ }^{43}$.

Qu'en était-il pour le caput viae marqué ab Augustobriga? Nous savons qu' Augustobriga était la première cité du conventus de Clunia du côté oriental, puisque la frontière entre les conventus de Clunia et de Caesaraugusta se situait entre son territoire qui dépendait de Clunia $^{44}$ et celui de Turiaso qui appartenait à Caesaraugusta ${ }^{45}$. Le jalonnement de la voie a donc été effectué seulement dans le conventus de Clunia. Signalons, en outre, une particularité qui caractérise tous les milliaires de Trajan recueillis dans le conventus de Clunia: sur aucun texte n' apparaît le nombre des puissances tribuniciennes de l'empereur ${ }^{46}$. Aussi pour cette voie Clunia-Augustobriga, comme à propos de celle de Galice qui a été examinée aupa-

39. E. Saavedra, op. cit., carte au $1 / 100000^{\mathrm{e}}$.

40. CIL II, 4896, 4899.

41. CIL II, 4890, 4893, 4894, 4898, 4900.

42. Le cas le plus explicite en Hispanie est celui de la Via Augusta de Bétique avec l' indication $a b$ arcu unde incipit Baetica sur des bornes de Domitien : P. Sillières, Les voies de communication..., op. cit., $\mathrm{n}^{\text {os }} 35$ et 41 .

43. Par exemple en péninsule Ibérique pour celle qui entrait dans Italica; d'après deux bornes d' Hadrien trouvées l' une à l' entrée de la ville et l' autre à quelques $\mathrm{km}$ au nord (P. Sillières, Les voies de communication..., op. cit. $\mathrm{n}^{\text {os }} 78$ et 79), elle était jalonnée par 25 bornes milliaires, distance qui menait sans doute à la frontière septentrionale de la cité car il n' y a, semble-t-il, aucune ville dans cette direction et à cette distance.

44. Augustobriga était une des cités des Pélendons (Ptolémée, II, 6, 53) peuple qui faisait partie du conventus de Clunia (Pline, HN, III, 27).

45. Pline, HN, III, 24.

46. J. Lostal Pros, Los miliarios de la provincia Tarraconense (conventus Tarraconense, Cesaraugustano, Cluniense y Cartaginense), Institución Fernando el Católico, Zaragoza 1992, 72. 
ravant, il paraît permis de supposer que c'est une autorité siégeant à Clunia, le chef-lieu du conventus, qui a décidé de son bornage.

Grâce à ces deux exemples, il nous semble maintenant très vraisemblable qu' un bureau chargé des voies se trouvait dans ces trois villes du pouvoir, chefs-lieux des circonscriptions administratives intermédiaires entre la province et les cités. En ce qui concerne les autres conventus de l' Hispanie, la documentation épigraphique n'est pas assez riche pour se prononcer. Il serait cependant assez étonnant que ces trois chefs-lieux de conventus aient fait exception et que les huit autres n' aient pas également été dotés d' un bureau des voies. Au moins tous ceux de la très vaste province d' Espagne citérieure dont le réseau des grandes chaussées était particulièrement développé et se prolongeait à de grandes distances de la capitale provinciale. 